\title{
Bootstrap method for constructing covariance matrices of optical-model parameters in the study of the threshold anomaly
}

\author{
Daniel Abriola ${ }^{\mathrm{a}}$, Guillermo V. Martí, and Jorge E. Testoni \\ Laboratorio TANDAR, Gerencia de Investigación y Aplicaciones, Comisión Nacional de Energía Atómica, B1650KNA San Martín, \\ Buenos Aires, Argentina
}

\begin{abstract}
The parameters of optical-model potentials are usually obtained by adjusting theoretical calculations to the corresponding experimental elastic-scattering data. It has been observed that the use of conventional covariance matrices for the evaluation of the uncertainties of the parameters obtained in this way, leads in general to unrealistically small values. This underestimate may be caused by either, an incorrect use of the statistical recipes, or by the lack of a systematic study of the robustness of the uncertainty values against the inclusion or exclusion of experimental data points within a given data set. In the present contribution we explore both factors. Regarding the first aspect we use a re-normalization for $\chi^{2}$, similar to the one proposed by R.T. Birge. In the second case we use the Bootstrap method to create synthetic sets based on all the available experimental data in order to derive an effective covariance matrix. These procedures were applied to the re-analysis of elastic-scattering data for several heavy-ion systems at energies close to the Coulomb barrier
\end{abstract}

\section{Introduction}

The simplest process in nuclear reactions is the elastic scattering of a projectile by a nuclear target. Angular distributions of elastically scattered ions have been of special interest in order to study the so-called threshold anomaly (TA). These angular distributions are adjusted with optical-model potentials either phenomenological, usually of a Wood-Saxon shape or semi-microscopic folding or double-folding potentials. Regarding the TA, two situations are considered: the dispersion of tightly bound or weakly bound projectiles. In the first case the TA is normally related to the closure of reaction channels as the bombarding energy decreases down to energies below the Coulomb barrier; this circumstance is reflected as a sudden decrease of the imaginary part of the optical-model potential and a corresponding appearance of a peak in the real part as function of bombarding energy [1]. For weakly bound projectiles, coupling to nonelastic channels (mainly the breakup-channel) generates a repulsive polarization potential that can produce either the absence of TA, or the so-called breakup threshold-anomaly (BTA). In this last case the imaginary potential increases while approaching the Coulomb barrier and, conversely, there is a reduction of the real part of the potential as a function of bombarding energy [2,3]. Theoretical models describing these nuclear-reaction processes lead to parameters that, according to their energy dependence, allow to determine the type of behavior in each case. An accurate evaluation of the model-parameter uncertainties is very important to decide which kind of anomaly corresponds to a determined set of experimental data, however, the used criteria for the calculations are often

a e-mail: abriola@tandar.cnea.gov. ar not so clear. It has been observed that the conventional covariance matrices for the evaluation of the uncertainties of the parameters lead frequently to unrealistically small values [4]. This underestimate most of the times is caused by an incorrect use of the statistical recipes. For instance, very often in the literature, one can find the use of the prescription of determining the parameter uncertainties in the minimization process, by requiring a variation of just one unit in the value of $\chi^{2}$. In cases where $\chi^{2} \gg v$, being $v$ the number of degrees of freedom, this recipe does not hold any more and has to be re-normalized [4]. Another aspect, perhaps more physical than mathematical, is the study of the effect of the exclusion of some experimental points and the change of the statistical weight of others, this procedure would simulate other possible experiments each one with a slightly different set of data. One objective of the present work is to explore both aspects. To study the first one, we perform a renormalization for $\chi^{2}$, following a method similar to the one proposed by R.T. Birge [5]. We use the Bootstrap method in order to explore the second approximation. The main idea of this method, applied in other fields but with little application in the analysis of nuclear reactions, is to create synthetic sets based on the available experimental data in order to derive an effective covariance matrix [4]. These procedures were applied to re-analyze elastic-scattering data of several heavy-ion systems at energies close to the Coulomb barrier. The experimental data were extracted from the EXFOR library [6]. Here, we report conclusions derived from the analyses of elastic-scattering differential crosssections from the ${ }^{12} \mathrm{C}+{ }^{208} \mathrm{~Pb}[7],{ }^{16} \mathrm{O}+{ }^{64} \mathrm{Zn} \mathrm{[8],}{ }^{6,7} \mathrm{Li}$ $+{ }^{27} \mathrm{Al}[9,10],{ }^{6,7} \mathrm{Li}+{ }^{80} \mathrm{Se}[11],{ }^{6} \mathrm{Li}+{ }^{64} \mathrm{Zn} \mathrm{[12],}{ }^{6} \mathrm{Li}$ $+{ }^{90} \mathrm{Zr}[13]$ and ${ }^{6,7} \mathrm{Li}+{ }^{144} \mathrm{Sm} \mathrm{[14]} \mathrm{systems.} \mathrm{The}{ }^{12} \mathrm{C}$ and ${ }^{16} \mathrm{O}$ projectiles are tightly bound whereas ${ }^{6,7} \mathrm{Li}$ are of the weakly bound type. 
Table 1. The first column is a number of order corresponding to the system in column two, the third column is the average number of experimental data points of all the angular distributions for the system, the fourth column is the reported $\left\langle\chi_{0}^{2}\right\rangle$ in the original reference, column five is the criterium used to evaluate the $N_{r}$ and $N_{i}$ parameters and the sixth column express the type of conclusion from the original work related to the TA or BTA. Columns seven and eigth are the percentage uncertainties in the $N_{r}$ and $N_{i}$ parameters given in the original references (see text). The column titled Covariance shows factors $f_{r}$ and $f_{i}$ that when multiplied by the original uncertainties $\left\langle\Delta N r_{\text {ref }}\right\rangle$ and $\left\langle\Delta N i_{\text {ref }}\right\rangle$ respectively would give the average uncertainties in this work, i.e., in system 1, $\left\langle\Delta N r_{\text {this-work }}\right\rangle=0.5\left\langle\Delta N r_{\text {ref }}\right\rangle=7.05 \%$ and similarly for the bootstrap factor.

\begin{tabular}{|c|c|c|c|c|c|c|c|c|c|c|c|}
\hline \multicolumn{8}{|c|}{ Original reference } & \multicolumn{2}{|c|}{$\begin{array}{c}\text { Covariance } \\
\text { factor }\end{array}$} & \multicolumn{2}{|c|}{$\begin{array}{c}\text { Bootstrap } \\
\text { factor }\end{array}$} \\
\hline & System & $\langle N\rangle$ & $\left\langle\chi_{0}^{2}\right\rangle$ & Crit. & TA Type & $\left\langle\Delta N r_{r e f}\right\rangle \%$ & $\left\langle\Delta N i_{\text {ref }}\right\rangle \%$ & $f_{r}$ & $f_{i}$ & $f_{r}$ & $f_{i}$ \\
\hline 1 & ${ }^{12} \mathrm{C}+{ }^{208} \mathrm{~Pb}$ & 24 & 6.1 & $2 * \chi_{0}^{2}$ & TA & 14.1 & 51.2 & 0.5 & 0.4 & 0.5 & 0.2 \\
\hline 2 & ${ }^{16} \mathrm{O}+{ }^{64} \mathrm{Zn}$ & 21 & 0.2 & $\chi_{0}^{2}+1$ & TA & 2.5 & 11.6 & 2.1 & 1.6 & 1.6 & 0.9 \\
\hline 3 & ${ }^{6} \mathrm{Li}+{ }^{27} \mathrm{Al}$ & 56 & 3.4 & $\chi_{0}^{2}+1$ & No TA & 4.7 & 8.8 & 1.4 & 1.5 & 1.4 & 1.1 \\
\hline 4 & ${ }^{7} \mathrm{Li}+{ }^{27} \mathrm{Al}$ & 49 & 4.2 & $\chi_{0}^{2}+1$ & No TA & 12.9 & 27.5 & 1.0 & 1.1 & 1.2 & 0.8 \\
\hline 5 & ${ }^{6} \mathrm{Li}+{ }^{80} \mathrm{Se}$ & 58 & 1.0 & $\chi_{0}^{2}+1$ & BTA & 4.0 & 7.1 & 1.3 & 1.8 & 0.8 & 1.0 \\
\hline 6 & ${ }^{7} \mathrm{Li}+{ }^{80} \mathrm{Se}$ & 49 & 1.0 & $\chi_{0}^{2}+1$ & TA & 4.8 & 11.3 & 1.1 & 1.6 & 0.9 & 0.7 \\
\hline 7 & ${ }^{6} \mathrm{Li}+{ }^{64} \mathrm{Zn}$ & 26 & 1.4 & $\chi_{0}^{2}+1$ & No TA & 16.0 & 7.6 & 0.3 & 0.8 & 0.2 & 0.5 \\
\hline 8 & ${ }^{6} \mathrm{Li}+{ }^{90} \mathrm{Zr}$ & 32 & 4.7 & $1.5 * \chi_{0}^{2}$ & No TA & 9.9 & 5.2 & 0.8 & 3.3 & 0.7 & 1.9 \\
\hline 9 & ${ }^{6} \mathrm{Li}+{ }^{144} \mathrm{Sm}$ & 50 & 2.7 & $\chi_{0}^{2}+1$ & BTA & 7.5 & 4.2 & 1.1 & 2.6 & 0.6 & 1.7 \\
\hline 10 & ${ }^{7} \mathrm{Li}+{ }^{144} \mathrm{Sm}$ & 50 & 1.4 & $\chi_{0}^{2}+1$ & No TA & 7.5 & 4.1 & 1.1 & 2.5 & 0.6 & 1.6 \\
\hline
\end{tabular}

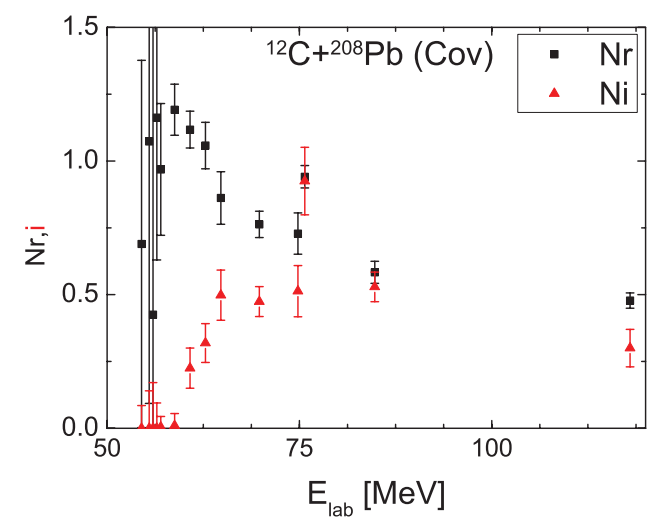

Figure 1. Parameters $\mathrm{Nr}$ and $\mathrm{Ni}$ for ${ }^{12} \mathrm{C}+{ }^{208} \mathrm{~Pb}$ and their uncertainties as function of bombarding energy, using the Covariance method.

\section{The covariance method}

In what follows we present results obtained using the above mentioned differential cross-sections, adjusting them with the São Paulo version of a double folding nuclear optical-model that delivers just two parameters, $\mathrm{Nr}$ and $\mathrm{Ni}$, related to the depth of the real and imaginary nuclear potential, respectively. In all cases the obtained parameters were indistinguishable from the values of the original references and with similar $\chi^{2}$ values. Two typical cases for TA and BTA are shown in Fig. 1 and Fig. 2 (TA) and in Fig. 3 and Fig. 4 (BTA) respectively.

We defined elliptic areas in the $\mathrm{Nr}-\mathrm{Ni}$ plane, that correspond to a confidence level of $68 \%$ this would implied a Mahalanobis distance of $2.3 \chi_{0}^{2} / \nu$. In the first of the above mentioned approximations, assuming a quadratic dependence of $\chi^{2}(\mathrm{Nr}, \mathrm{Ni})$, we obtain the blue ellipses of Fig. 5 and Fig. 6, defined by the following equation [4]: $2.3 \frac{\chi_{0}^{2}}{v}=\left(N r-N r_{0}, N i-N i_{0}\right)^{T} C^{-1}(N r-$ $N r_{0}, N i-N i_{0}$ ), where $C^{-1}$ is the inverse of the covariance matrix. In the second case, by means of sets of

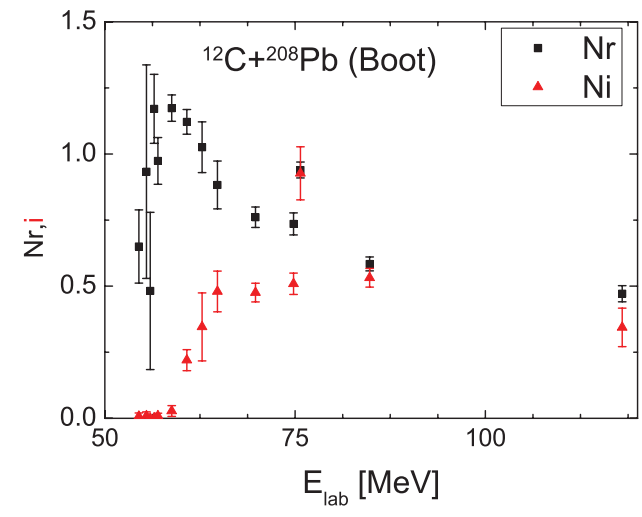

Figure 2. Parameters $\mathrm{Nr}$ and $\mathrm{Ni}$ for ${ }^{12} \mathrm{C}+{ }^{208} \mathrm{~Pb}$ and their uncertainties as function of bombarding energy, using the Bootstrap method.

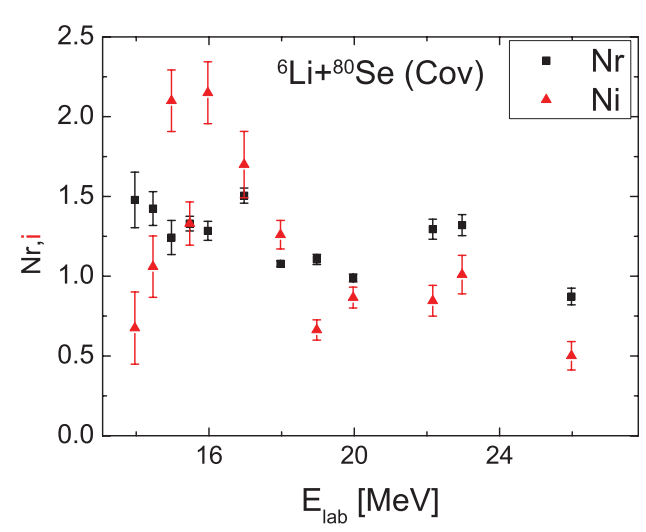

Figure 3. Parameters $\mathrm{Nr}$ and $\mathrm{Ni}$ for ${ }^{6} \mathrm{Li}+{ }^{80} \mathrm{Se}$ and their uncertainties as function of bombarding energy, using the Covariance method.

statistical achieved best fits obtained through the Bootstrap procedure, as explained in the next section, we obtained the gray points and red ellipses shown in the same figures. 


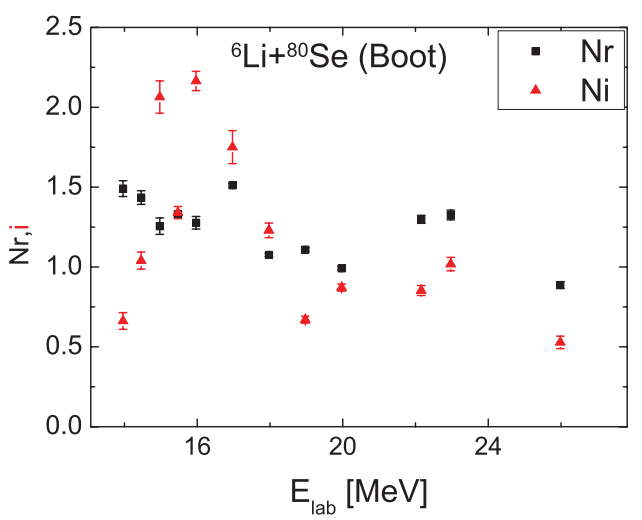

Figure 4. Parameters $\mathrm{Nr}$ and $\mathrm{Ni}$ for ${ }^{6} \mathrm{Li}+{ }^{80} \mathrm{Se}$ and their uncertainties as function of bombarding energy, using the Bootstrap method.

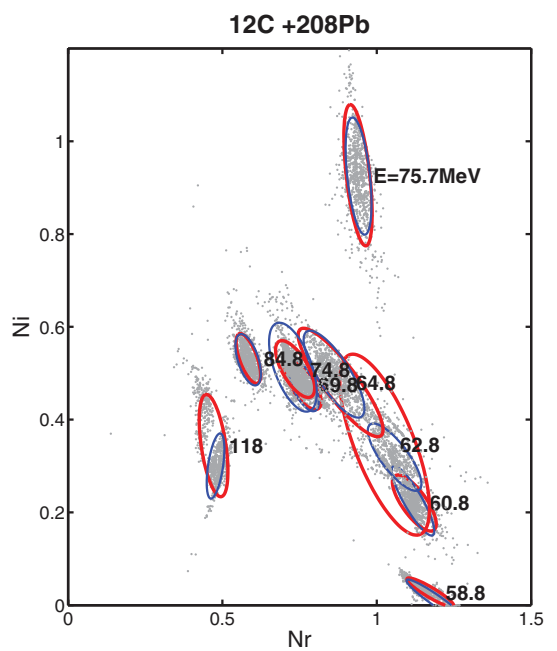

Figure 5. Covariance (blue) and bootstrap ellipses (red) for the ${ }^{12} \mathrm{C}+{ }^{208} \mathrm{~Pb}$ system. The gray points are the individual bootstrapped $\mathrm{Nr}$ and $\mathrm{Ni}$ values.

\section{The Bootstrap method}

Bootstrap is one of the many re-sampling methods designed to go beyond regular statistic test. It creates a number NB of synthetic data sets, each of them consisting on $\mathrm{N}$ data points selected randomly (with reposition) from the original data set of $\mathrm{N}$ points (it is desirable to have $\mathrm{NB} \gg \mathrm{N}$ ). On the average a fraction 1/e (about 37\%) of the $\mathrm{N}$ points will be repeated ones (thus having more weight in the fitting procedure) and, consequently, 37\% of the elements will not be included. Each synthetic data set is used to fit the parameters of interest. The standard deviation of the NB values obtained for each parameter is a reliable estimation of its uncertainty. Hence, this procedure gauges the sensitivity of the fitted parameter to each individual data point by the simulation of NB experiments in which the experimenter could have chosen to repeat the measurement of some of the data points at the cost of missing some others. To the best of our knowledge, Bootstrap techniques have not been yet applied to the calculation of nuclear potential parameters and their uncertainties. In Figs. 5 and 6 we show the resulting $\mathrm{Nr}$, $\mathrm{Ni}$ points from the adjustment of $\mathrm{NB}=1000$ synthetic angular distributions. The average and variance converged for $\mathrm{NB} \geq 200$. It is interesting to note the two cases of Fig. 5 in which the ellipses (the red and blue ones) differ

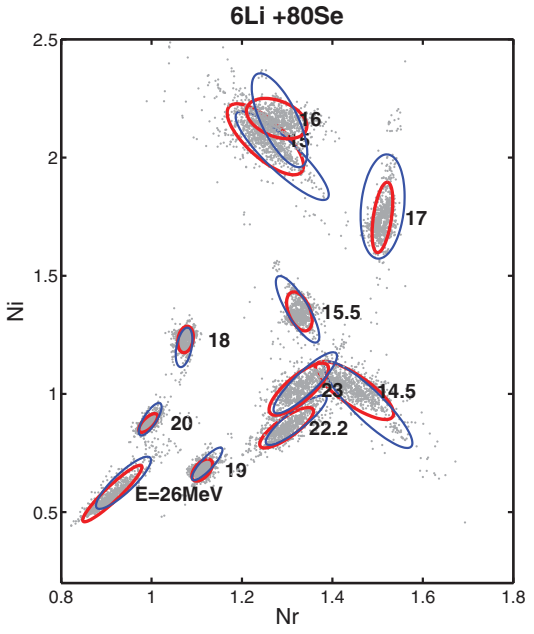

Figure 6. Covariance (blue) and bootstrap ellipses (red) for the ${ }^{6} \mathrm{Li}+{ }^{80} \mathrm{Se}$ system. The gray points are the individual bootstrapped $\mathrm{Nr}$ and $\mathrm{Ni}$ values.

significantly, they correspond to those labeled $118 \mathrm{MeV}$ and $63 \mathrm{MeV}$, which are the two energies with the lowest number of experimental data points for that system (19 and 11 points respectively). The bootstrap technique explores the consequences of changing the statistical weight of each point in the angular distributions. In these two cases the bootstrap ellipses produced larger uncertainties than the covariance method, showing enough sensitivity to evidence outlier points or unrealistic uncertainties in the original experimental data.

\section{Conclusions}

The Bootstrap method produced central values of parameters identical (within 1\%) with the covariance method and uncertainties somewhat smaller. Our analysis indicates that the uncertainties of the parameters in the original works for systems 1 and 7 should be reduced by a factor close to two (see Table 1), the uncertainties of system 4 remains approximately the same while for all the other systems it should be increased. Even though most of the parameter uncertainties experimented changes, these changes did not alter the conclusions related to the type of TA. The Bootstrap method allows the construction of an effective covariance matrix, and related uncertainties, without performing derivatives in the minimum of the multidimensional $\chi^{2}$-surface, thus avoiding different problems like validity of quadratic models, numerical problems, or difficulties in the minimization procedure. In the particular cases of data set with a small number of points, the bootstrap method allows the detection of outliers in the experimental angular distributions or points with unrealistic experimental uncertainties; thus producing parameters with larger and more robust uncertainties. In a further work we plan to applied the method to other nuclear reaction systems.

\section{References}

[1] G.R. Satchler, Physics Report 199, 147 (1991)

[2] M. Hussein, et al. Physical Review C73, 044610 (2006)

[3] M. Hussein, et al. Physical Review C76, 019902(E) (2007) 
[4] D. Abriola, et al. Journal of Physics: Conference Series 630, 012021 (2015)

[5] R.T. Birge, Phys. Rev. 40, 207 (1932)

[6] Nuclear Data Sheets 120, 272 (2014)

[7] S. Santra, et al. Physical Review C64, 024602 (2001)

[8] C. Tenreiro, et al. Physical Review C53, N06 2870 (1996)

[9] J. Fernández Niello, et al. Nuclear Physics A787, 484c (2007)
[10] J.M. Figueira, et al. Physical Review C73, 054603 (2006)

[11] L. Fimiani, et al. Physical Review C86, 044607 (2012)

[12] M. Zadro, et al. Physical Review C80, 064610 (2009)

[13] H. Kumawat, et al. Physical Review C78, 044617 (2008)

[14] J.M. Figueira, et al. Physical Review C81, 024613 (2010) 